\title{
Ultrasound-based assessment of hyomental distances in neutral, ramped, and maximum hyperextended positions, and derived ratios, for the prediction of difficult airway in the obese population: a pilot diagnostic accuracy study
}

\author{
Cristina Petrișor ${ }^{1,2}$, Robert Szabo ${ }^{1}$, Cătălin Constantinescu ${ }^{1}$, Adrian Prie ${ }^{1}$, Natalia Hagău $u^{1,2}$ \\ 1"Iuliu Hațieganu" University of Medicine and Pharmacy Cluj-Napoca, Romania \\ ${ }^{2}$ The Clinical Emergency County Hospital Cluj, Romania
}

\begin{abstract}
Background: Ultrasonography-assessed hyomental distance (HMD) ratio has been found to discriminate between obese patients with Cormack-Lehane grades 1 or 2 vs. those with grades 3 or 4 . The aim of our study is to evaluate the performance of the HMD evaluated ultrasonographically in neutral, ramped, and maximum hyperextended positions, as well as for the ratios obtained by dividing the HMD in the ramped position to that in the neutral position (HMDR1) and by dividing the HMD in maximum hyperextension to that in the neutral position (HMDR2), in order to predict the occurrence of Cormack-Lehane grades 3 or 4 during direct laryngoscopy.

Methods: Ultrasound measurements were performed preoperatively in 25 patients with morbid obesity, measuring the HMD in the neutral, ramped, and maximum hyperextended positions. Pre-epyglotic soft tissue thickness, Mallampati and upper lip bite test scores were recorded. Cormack-Lehane grading was considered as an outcome variable in the Receiver Operating Characteristics curve analysis.

Results: HMD in the neutral, ramped and maximum hyperextended positions presented similar sensitivities, $100 \%$ [39.8-100.0]. For HMD, specificity was 42.86\% [21.8-66.0] in the neutral position, 61.9\% [38.4-81.9] in the ramped postion, and $71.4 \%$ [47.8-88.7] in the maximum hyperextended position. For HMDR1, the cut-off value was 1.12. This threshold provides 75\% [19.4-99.4] sensitivity and 76.2\% [52.8-91.8] specificity. For HMDR2, a cut-off value of 1.23 provides $100 \%$ [39.8-100.0] sensitivity and 90.5\% [69.6-98.8] specificity.

Conclusion: HMDR2 seems to have superior diagnostic accuracy in predicting difficult laryngoscopy in the obese population compared to HMDR1, as well as compared to the HMD in the neutral, ramped, and maximum hyperextended positions.
\end{abstract}

Anaesthesiology Intensive Therapy 2018, vol. 50, no 2, 110-116

Key words: airway assessment, ultrasonography; difficult airway, difficult laryngoscopy

The prediction of difficult airway is of utmost importance for anaesthesiologists, intensivists and emergency physicians. Inability to anticipate difficult laryngoscopy and difficult intubation can have dramatic consequences, which leads to an endless search for a screening tool that has good sensitivity and specificity, is reliable and easy to use, and is also reproducible. This is even more important in obese patients, who have a higher incidence of difficult airways compared to the general population [1]. Ultrasonography, being a non-invasive, cheap and non-irradiating technique, has gained widespread use among anaesthesiologists. For difficult airway prediction in the obese population, Wojtczak et al. [2] found that the ultrasonographic measurements of the hyomental distance (HMD) in a hyperextended position and the hyomental distance ratio (HMDR), obtained by dividing the HMD in maximum hyperextension to that 
in the neutral position, differentiates between obese patients with Cormack-Lehane grades 1 or 2 versus those with grades 3 or 4 .

The aim of the study is to evaluate the performance of the hyomental distances evaluated ultrasonographically in the neutral, ramped, and maximum hyperextended positions, as well as for the ratios obtained by dividing the HMD in the ramped position to that in the neutral position (HMDR1) and by dividing the HMD in maximum hyperextension to that in the neutral position (HMDR2), in order to predict the occurrence of Cormack-Lehane grades 3 or 4 during direct laryngoscopy. By conducting a Receiver Operating Characteristics (ROC) curve analysis to investigate the diagnostic accuracy of these parameters, the optimal threshold with the most discriminative power can be identified that confers the best sensitivity and specificity for each of the above ultrasonographically measured screening parameters.

\section{METHODS}

The approval for performing preoperative ultrasound and including patients in the study was obtained from the Ethics Committee of the Clinical Emergency County Hospital, Cluj-Napoca, Romania (No.18637/20.09.2016). All patients signed the informed consent form. Patients with morbid obesity (Body Mass Index $>40 \mathrm{~kg} \mathrm{~m}^{-2}, \mathrm{BMI}$ ), who needed to be operated on under general anaesthesia with oro-tracheal intubation, were included. Patients in whom the use of a videolaryngoscope was planned or who required rapid sequence induction with external laryngeal manipulation were excluded from the study. In total, thirty-one potential eligible patients were considered for inclusion in this pilot diagnostic accuracy study. Two patients were excluded due to their refusal to participate and four were excluded due to the use of a videolaryngoscope on the first attempt to intubate. Thus, the ultrasound screening tests for difficult laryngoscopy were run on 25 eligible patients one day prior to the surgical intervention. The Mallampati, the upper lip bite test (ULBT) and BMI scores were recorded. Mallampati grades 3 and 4 , as well as ULBT grade 3, were considered to be difficult airway $[3,4]$.

Ultrasound scanning of the submandibular region was performed with a curvilinear transducer (Venue 50 Sonograph, General Electrics, Fairfield, Connecticut, USA). In the suprahyoid region, a midsaggital longitudinal scan reveals the hypoechoic muscles forming the floor of the mouth inbetween two hyperechoic bony structures that have a posterior acoustic shadowing: the hyoid bone and the posterior aspect of the symphisis menti $[5,6]$. We measured the HMD distance between the hyoid bone and the posterior border of the symphisis menti in three positions, namely: neutral, ramped, and maximum hyperextended (Fig. 1).
For the neutral position, the patient was placed supine and asked to look straight forward. For the ramped position, the patient was placed so that the external auditory meatus was on a horizontal line with the sternum. For the maximum hyperextended position, the patient was asked to hyperextend the head back as much as possible. We defined HMDR1 (hyomental distance ratio 1 ) as the ratio between the HMD in the ramped position to that in neutral position, and HMDR2 (hyomental distance ratio 2 ) as the ratio between the HMD in the maximum hyperextended position to that in the neutral position.

The pre-epyglotic soft tissue thickness was measured using a linear transducer at the level of the vocal cords.

The laryngoscopy view was obtained by performing a direct laryngoscopy with a Macintosh blade in patients in whom anaesthesia induction was performed in the ramped position, with complete muscle paralysis. The view was graded by anaesthesiologists with 7-30 years of practice. The Cormack-Lehane scoring system was used as the outcome variable, patients with grades 1 or 2 being considered the easy laryngoscopy group, and grades 3 or 4 being considered as the difficult laryngoscopy group [7].

Cut-off values for all ultrasound-derived index tests were obtained from ROC curves [8]. The accuracy of the tests, using the AUC, was evaluated. The sensitivity and specificity of each parameter were compared. For Mallampati and ULBT tests, sensitivities and specificities were calculated using a 2 $\times 2$ contingency table.

\section{RESULTS}

During laryngoscopy, four patients (15.8\%) presented Cormack-Lehane grades 3 or 4 and were labelled as the difficult laryngoscopy group, while the rest of the patients (84.2\%) had Cormack-Lehane grades 1 or 2 and were considered as the easy laryngoscopy group (Table 1). No statistical differences were found between the patients at the baseline.

Data are expressed as mean values, with standard deviation in parentheses; HMD: hyomental distance; HMDR1: the ratio of the hyomental distance in the ramped position and that in the neutral position; HMDR2: ratio between the hyomental distance in the maximum hyperextended position to that in the neutral position

Between the difficult and easy laryngoscopy groups, the mean HMD in the ramped and maximum hyperextended positions differed significantly $(P=0.031$, and $P=0.015$, respectively). pThe same was observed when comparing HMDR1 $(P=0.024)$ and HMDR2 $(P=0.0002)$. No difference was found when comparing the HMD in the neutral position between the two groups $(P=0.316)$ (Table 1$)$.

We investigated the performance of the HMD in different positions for the prediction of the occurrence of difficult 

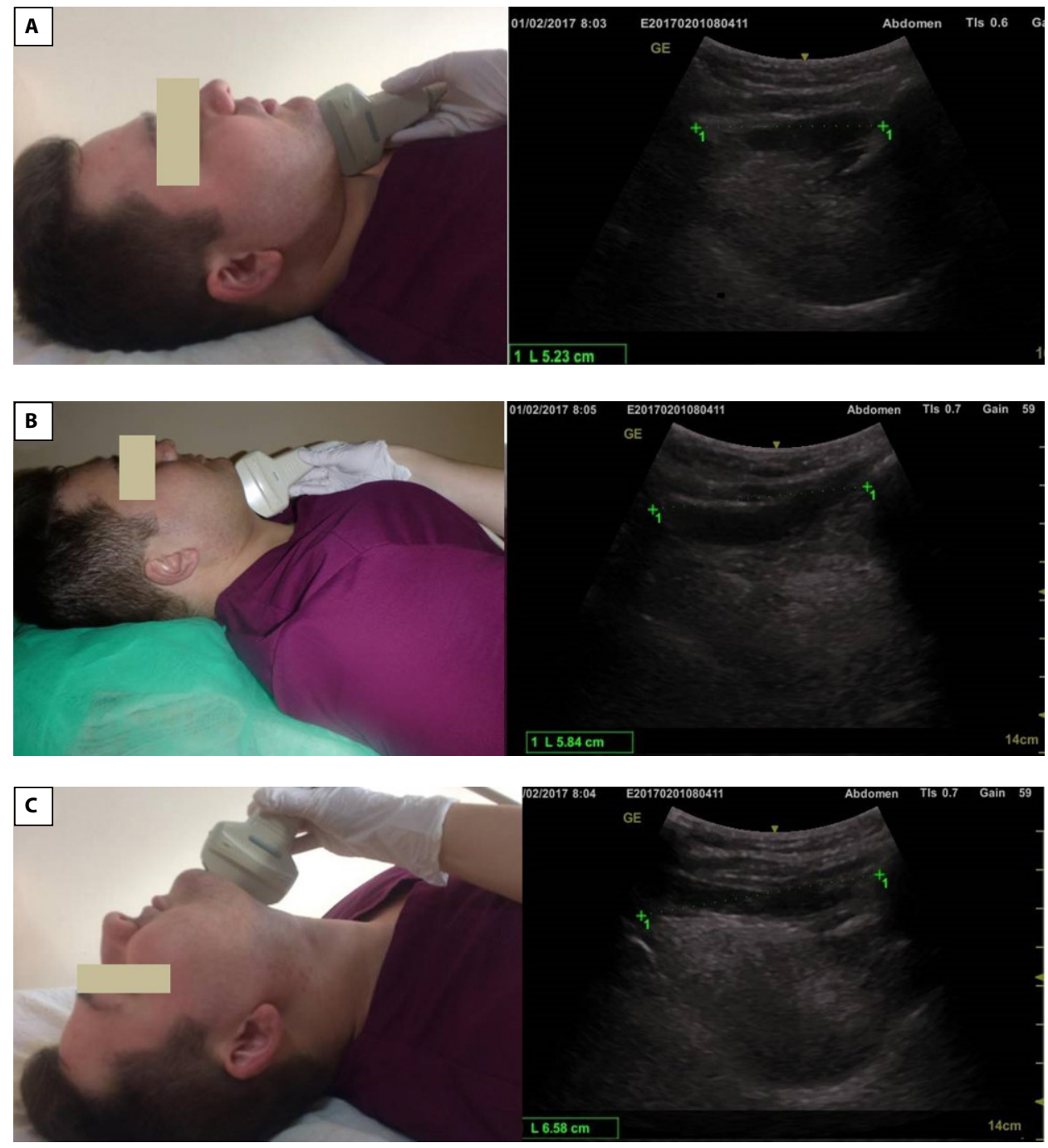

Figure 1. Hyomental distance ultrasound measurements in neutral (A), ramped (B), and maximum hyperextended positions (C)

Table 1. Demographical and clinical data of patients divided into difficult and easy laryngoscopy groups

\begin{tabular}{lccc}
\hline & $\begin{array}{c}\text { Difficult laryngoscopy } \\
\text { (Cormack-Lehane 3 and 4) }\end{array}$ & $\begin{array}{c}\text { Easy laryngoscopy } \\
\text { (Cormack-Lehane 1 and 2) }\end{array}$ & $\begin{array}{c}\text { P-value } \\
\text { No. }\end{array}$ \\
Sex (male:female) & 4 & 21 & - \\
Age (years) & $1: 3$ & $8: 13$ & - \\
Body mass index (kg m-2) & $52(12)$ & $46(14)$ & 0.43 \\
Mallampati test & $43.97(7.55)$ & $45.58(3.79)$ & 0.7 \\
Upper lip bite test & $3.5(0.57)$ & $2.04(0.74)$ & 0.38 \\
Pre-epyglotic soft tissue thickness (mm) & $2(0.81)$ & $1.38(0.58)$ & 0.15 \\
HMD neutral $(\mathrm{cm})$ & $15.75(30.73)$ & $17.39(15.15)$ & 0.6 \\
HMD ramped $(\mathrm{cm})$ & $4.04(0.1)$ & $4.34(0.32)$ & 0.31 \\
HMD maximum $(\mathrm{cm})$ & $4.53(0.1)$ & $5.17(0.28)$ & 0.03 \\
HMDR1 & $4.9(0.22)$ & $5.8(0.42)$ & 0.01 \\
HMDR2 & $1.12(0.001)$ & $1.2(0.01)$ & 0.02 \\
\hline
\end{tabular}




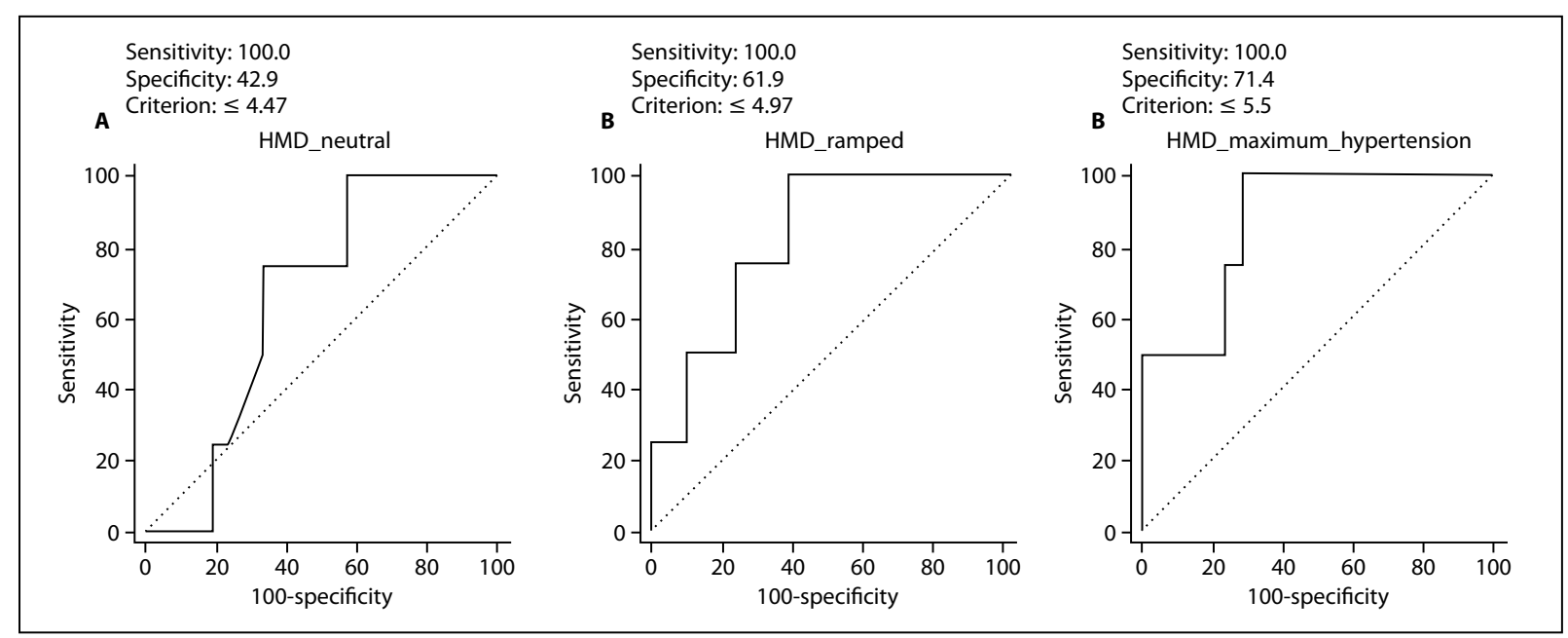

Figure 2. ROC curves for the performance of hyomental distances (HMD) measured in neutral (HMD_neutral), ramped (HMD_ramped), and maximum hyperextended (HMD_maximum_hyperextended) positions

Table 2. Receiver Operating Characteristics $(\mathrm{ROC})$ curve analysis for investigated parameters

\begin{tabular}{|c|c|c|c|c|c|}
\hline Parameter & Cutoff & Sensitivity & Specificity & AUC & $P$ \\
\hline HMD_neutral (cm) & $\leq 4.47$ & $100[39.8-100]$ & $42.86[21.8-66]$ & $0.66[0.44-0.83]$ & 0.17 \\
\hline HMD_ramped $(\mathrm{cm})$ & $\leq 4.97$ & $100[39.8-100]$ & 61.9 [38.4-81.9] & $0.82[0.62-0.94]$ & 0.0007 \\
\hline HMD_maximum (cm) & $\leq 5.5$ & $100[39.8-100]$ & $71.4[47.8-88.7]$ & $0.87[0.67-0.97]$ & $<0.0001$ \\
\hline Pre-epyglotic soft tissue (mm) & $\leq 13.8$ & $75[19.4-99.4]$ & $75[50.9-91.3]$ & $0.63[0.41-0.82]$ & 0.49 \\
\hline HMDR1 & $\leq 1.12$ & $75[19.4-99.4]$ & $76.2[52.8-91.8]$ & $0.75[0.54-0.90]$ & 0.037 \\
\hline HMDR2 & $\leq 1.23$ & $100[39.8-100]$ & 90.5 [69.6-98.8] & $0.92[0.73-0.98]$ & $<0.0001$ \\
\hline
\end{tabular}

HMD: hyomental distance, HMDR1: the ratio of the hyomental distance in the ramped position and that in the neutral position; HMDR2: ratio between the hyomental distance in the maximum hyperextended position to that in the neutral position; AUC: area under the curve for ROC curve analysis; $P$-value for the ROC curve analysis

view during direct laryngoscopy (Fig. 2), as well as that of the pre-epyglotic soft tissue thickness.

The HMD in the neutral, ramped and maximum hyperextended positions presented similar sensitivities, 100\% [39.8-100]. For the HMD in the neutral position, specificity was $42.86 \%$ [21.8-66.0]. The specificity of the HMD increased to $61.9 \%$ [38.4-81.9] when the patient was placed in the ramped position, and to $71.4 \%$ [47.8-88.7] in the maximum hyperextended position (Table 2). For the pre-epyglotic soft tissue thickness, the ROC curve analysis indicated a cut-off value of $13.8 \mathrm{~mm}$, with sensitivity and specificity of approximately $75 \%$.

The AUCs for the HMD in the maximum hyperextended and ramped positions were significantly higher than that of the HMD measured in the neutral position $(P=0.0354$ for the HMD neutral vs. ramped and $P=0.0017$ for HMD neutral vs. maximum positions), while there were no significant differences between the AUCs of the ROC curve for the HMD in ramped vs. maximum hyperextended positions $(P=0.51)$.

For HMDR1, the cut-off value was 1.12, with an AUC $0.75(P=0.0378)$. This threshold provides 75\% [19.4-99.4] sensitivity and 76.2\% [52.8-91.8] specificity (Table 2 ).
For HMDR2, a cutoff value of 1.23 provides $100 \%$ [39.8-100] sensitivity and 90.5\% [69.6-98.8] specificity, the highest values of all the investigated parameters (Table 2). Thus, the ability of the patient to expand the distance between the hyoid bone and simphysis menti by more than $23 \%$ from the neutral to maximum hyperextended positions, allowed us to rule out difficult laryngoscopy in $90.5 \%$ of the patients in the easy laryngoscopy group. The inability to expand this distance by more than $23 \%$, allowed us to identify $100 \%$ of the patients with difficult views during direct laryngoscopy.

There was no significant statistical difference between the AUCs resulting from the ROC curve analysis for HMDR1 and HMDR2 ( $P=0.0968$ ) (Fig. 3). Although the AUC for HMDR2 was significantly higher than that of the HMD in the neutral position ( $P=0.046)$, there were no differences between the AUCs for HMDR2 vs. HMD in the ramped position or maximum hyperextension ( $P$-values 0.33 , and 0.54 , respectively), nor between the AUC of HMDR1 and all the other investigated variables (HMDR1 vs. HMD neutral $P=0.35$, vs. HMD ramped $P=0.63$, and vs. HMD maximum hyperextension $P=0.30$ ).

In our cohort of patients, for the Mallampati test we found $100 \%$ [39.76-100] sensitivity and $71.43 \%$ 


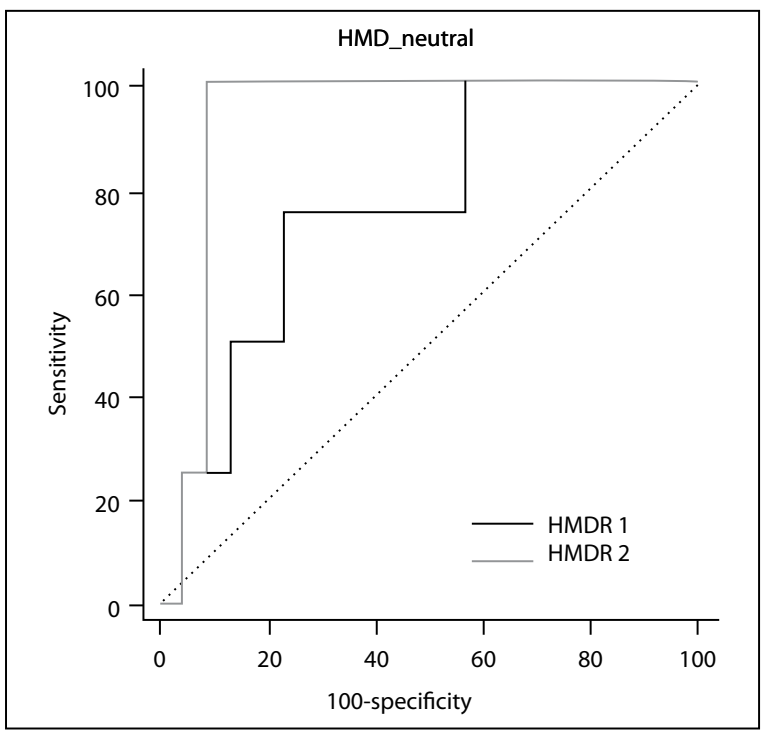

Figure 3. ROC curve analysis for HMDR1 and HMDR2 for the prediction of the occurrence of Cormack-Lehane grades 3 or 4 during direct laryngoscopy

[47.82-88.72] specificity, while for the ULBT test we found $25 \%[0.63-80.59]$ sensitivity and $100 \%$ [83.89-100] specificity. HMDR1 and HMDR2 were superior in terms of performance compared to the ULBT (McNemar test, $P=0.023$ and 0.0412 , respectively), but were not superior to Mallampati score (McNemar test, $P=0.77$ and 0.13 , respectively).

\section{DISCUSSION}

The inability to successfully intubate and ventilate a patient after anaesthesia induction can lead to catastrophic results, including severe hypoxic brain damage, and even death. Clinical predictors are not $100 \%$ reliable to anticipate difficult laryngoscopy and difficult intubation. Currently available quantifiable clinical tests that predict difficult intubation are the Mallampati classification and the ULBT test, although both seem to be poor predictors as single screening tests $[4,9,10]$. Even when we combine clinical tests to obtain an integrated score, they yield a low sensitivity and specificity and take long to perform. This is in part due to the subjective nature of some tests, the inability of the patient to understand or perform the test and due to incorrect identification of anatomical structures [11]. The latter may be amplified in obese patients. Any clinical tool that can improve airway management is a welcome addition to clinical assessment [12].

When evaluating diagnostic tests a high sensitivity and specificity is desired, with the former being of greater importance [13]. Ultrasonography-derived indicators have been recently evaluated for the prediction of difficult airway. However, data on the impact of US use in airway management remain limited [14]. In the non-obese population, the ultrasonographic measurements of the distance from skin to epiglottis, the ability or inability to visualise the hyoid bone by performing sublingual sonography, and tongue thickness, have been correlated with Cormack-Lehane scores [15-20]. Similarly, in the obese population, the pretracheal soft tissue thickness has been linked to difficult airway [21].

In a small cohort of 5 obese and 7 morbidly obese patients, the hyometal distance ratio differentiated between patients with difficult intubation and with easy intubation, thus HMDR may predict difficult laryngoscopy that can result in difficult intubation [2]. Moreover, the HMD in the hyperextended position also differed significantly in the two groups. These parameters, obtained by performing submandibular sonography, have not yet been assessed while their performance has not been established, in terms of sensitivity and specificity, in STARD-compliant studies.

We measured HMD in the neutral, ramped, and maximum hyperextended positions and defined HMDR1 and HMDR2. The sensitivity, specificity and AUC of each diagnostic test were calculated using ROC curves analysis. To our knowledge, no such study has been conducted on obese patients to identify the optimal threshold that confers the best sensitivity and specificity to anticipate a difficult view during direct laryngoscopy.

Our ROC curve analysis revealed that HMDR2 seems to have superior diagnostic accuracy than all the investigated indicators we measured using ultrasonography, even though statistical significance has not been achieved. With an incidence of difficult intubation of $15.8 \%$ and an optimal threshold of 1.23 , sensitivity was $100 \%$ and specificity $90.48 \%$. Although the incidence of Cormack-Lehane grades 3 or 4 during direct laryngoscopy is around $5.8 \%$ in nonobese patients, in the obese population this percentage can be as high as $15.4 \%[1,10]$. The incidence of difficult view during direct laryngoscopy in our cohort is comparable with this. Highly sensitive tests ensure that false negative results are identified and failed intubations prevented. If we consider the incidence of Cormack 3 or 4 in the obese population is around $15 \%$, with 0.8 power and an alpha of 0.05 , a number of 20 patients is required to prove that the AUC for HMDR2 is approximately 0.9, a fact that we have demonstrated in this study [22]. However, to prove that HMDR2 has an AUC of 0.9, while that of HMDR1 is 0.75 , and thus accuracy is lower, a number of 2297 patients would be required [22]. It is possible that HMDR2 provided the highest performance of all investigated variables as it reflects the maximum anterior displacement of the mandible, which is achieved during direct laryngoscopy.

HMD in maximal hyperextension, HMDR1 and HMDR2 may be indicators of the compliance of the submandibular space. They reflect the possibility to displace the mandible anteriorly and to expand the distance between the hyoid 
bone and the mandible. In patients with obesity, the compliance is decreased by the deposition of fat in the anterior neck region. The elasticity in saggital plane is reflected in the expansion of the HMD from the neutral to the ramped, and then to the maximum hyperextended position. This is the equivalent of the hyo- incisor distance, one of the components of the tridimensional model of Greenland et al. [23] for submandibular space compliance. HMDR2 is also affected by neck mobility.

The hyomental distances ratio obtained by dividing the hyomental distance in the hyperextended postion to that in the neutral position, measured clinically from the tip of the chin to the palpable hyoid bone, was evaluated in the non-obese population. With a cutoff of 1.2 , this clinical HMDR yielded $88 \%$ sensitivity and $60 \%$ specificity [24]. This cutoff is close to our ultrasonography HMDR2. However, in obese patients, the hyoid bone is more difficult to palpate. Ultrasonography confers precision. In this study, in order to increase accuracy, all distances were measured using ultrasound, and HMDR ratios were calculated based on these values. This method is superior to clinical examination as it offers precise anatomic information which would not be obvious otherwise, as measurements are expressed in millimetres. Ultrasound is comparable to computerized tomography and magnetic resonance imaging when used to evaluate upper airway and, at the same time, is faster, cheaper and non-invasive [25]. For scanning, we used the method described by Wojtczak et al. [2], which forms a focused point-of-care ultrasonography algorithm of the airway $[2,26]$.

Andruszkiewicz et al. [27] evaluated several sonographic measurements in predicting difficult laryngoscopy in 199 patients without morbid obesity and concluded that individual measurements have unsatisfactory predictive roles, HMDR having only $43 \%$ sensitivity and $96 \%$ specificity. However, morbidly obese patients have a different airway anatomy, different submandibular space compliance characteristics and a different standard position (ramped) during direct laryngoscopy. Thus, the performance of ultrasound-derived HMDR might differ in morbidly obese patients compared with the same predictive technique for non-obese patients.

The technique may be used by anaesthesiologists, emergency and intensive care physicians. Even though it is well known that ultrasonography is operator dependant, the scanning technique is simple and the anatomical landmarks represented by the hyoid and the mandible do not allow room for misinterpretations. Moreover, the view obtained during direct laryngoscopy is dependent on patient position, which should be ramped for the obese, as well as on good muscle paralysis and on the skills of the anaesthesiologists. We overcame these in our study by providing muscle relaxation in each patient and involving skilled anaesthesi- ologists. For patient positioning, the three axis alignment theory is still valid: non-obese patients are placed in the standard sniffing position, while in obese patients, a ramped position should be used to achieve adequate exposure of the larynx [28]. All our patients were placed in the ramped position before measuring the HMD, which we consider more appropriate when evaluating obese patients when compared with the classic supine position. In studies investigating airway difficulty, the outcome variable is always the Cormack-Lehane score, even though difficult view during direct laryngoscopy is not necessarily equal to difficult intubation [29]. We have also used Cormack-Lehane grading as the main outcome.

Of all investigated parameters, including the pre-epyglotic soft tissue thickness, HMDR2 seems to have the highest diagnostic accuracy, although we cannot yet say that it is significantly superior to the other parameters from a statistical point of view. Larger studies are required to prove this assumption. A comparison of HMDR2 with other ultrasonographic indicators, as well as current standard clinical screening tests is necessary in order to establish the value of HMDRs in clinical practice.

\section{CONCLUSIONS}

The ratio of the HMD in the maximum hyperextended position to that in the neutral position seems to have superior diagnostic accuracy in predicting difficult laryngoscopy in the obese population compared with the ratio of the HMD in the ramped position to that in the neutral position, as well as compared with HMD in the neutral, ramped, and maximum hyperextended positions. With an optimal threshold of 1.23, this parameter has an AUC of 0.92 [0.73-0.98] $(P<$ $0.0001), 100 \%$ [39.8-100] sensitivity, and 90.5\% [69.6-98.8] specificity for the anticipation of Cormack-Lehane grades 3 or 4 in the obese population.

\section{ACKNOWLEDGEMENTS}

1. Source of funding: none.

2. Conflict of interest: none.

\section{References:}

1. Budde $A O$, Desciak M, Reddy V, et al. The prediction of difficult intubation in obese patients using mirror indirect laryngoscopy: A prospective pilot study. J Anaesthesiol Clin Pharmacol. 2013; 29(2): 183-186, doi: 10.4103/0970-9185.111685, indexed in 9185 .

2. Wojtczak JA. Submandibular sonography: assessment of hyomental distances and ratio, tongue size, and floor of the mouth musculature using portable sonography. J Ultrasound Med. 2012; 31(4): 523-528, indexed in Pubmed: 22441908.

3. Khan ZH, Kashfi A, Ebrahimkhani E. A comparison of the upper lip bite test (a simple new technique) with modified Mallampati classification in predicting difficulty in endotracheal intubation: a prospective blinded study. Anesth Analg. 2003; 96(2): 595-9, table of contents, indexed in Pubmed: 12538218. NCBI. SpringerReference doi: 10.1007/ springerreference_36064.

4. Lundstrøm LH, Vester-Andersen M, Møller AM, et al. Danish Anaesthesia Database. Poor prognostic value of the modified 
Mallampati score: a meta-analysis involving 177088 patients. $\mathrm{Br} \mathrm{J}$ Anaesth. 2011; 107(5): 659-667, doi: 10.1093/bja/aer292, indexed in Pubmed: 21948956.

5. Choudhary G, Boparai A, Singh G, et al. Value of ultrasound in understanding laryngeal anatomy: pictorial review. Pakistan Journal of Radiology. 2016; 22(1): 4-7.

6. Singh M, Chin KiJ, Chan VWS, et al. Use of sonography for airway assessment: an observational study. J Ultrasound Med. 2010; 29(1): 79-85, indexed in Pubmed: 20040778.

7. Cormack RS, Lehane J. Difficult tracheal intubation in obstetrics. Anaesthesia. 1984; 39(11): 1105-1111, indexed in Pubmed: 6507827.

8. Zhou XH, Obuchowski NA, McClish DK. Statistical Methods in Diagnostic Medicine. JohnWiley: New York, 2002.

9. Eberhart LHJ, Arndt C, Cierpka T, et al. The reliability and validity of the upper lip bite test compared with the Mallampati classification to predict difficult laryngoscopy: an external prospective evaluation. Anesth Analg. 2005; 101(1): 284-9, table of contents, doi: 10.1213/01. ANE.0000154535.33429.36, indexed in Pubmed: 15976247.

10. Shiga T, Wajima $Z$, Inoue $T$, et al. Predicting difficult intubation in apparently normal patients: a meta-analysis of bedside screening test performance. Anesthesiology. 2005; 103(2): 429-437, indexed in Pubmed: 16052126

11. Gupta S, Sharma R, Jain D. Airway assessment: predictors of difficult airway. Indian J Anaesth. 2005; 49(4): 257-262.

12. Teoh WH, Kristensen MS. Utility of ultrasound in airway management. Trends in Anaesthesia and Critical Care. 2014; 4(4): 84-90, doi: 10.1016/j. tacc.2014.05.004

13. Healy DW, LaHart EJ, Peoples EE, et al. A Comparison of the Mallampati evaluation in neutral or extended cervical spine positions: a retrospective observational study of $>80000$ patients. Br J Anaesth. 2016; 116(5): 690-698, doi: 10.1093/bja/aew056, indexed in Pubmed: 27106973.

14. Kundra P, Mishra SK, Ramesh A. Ultrasound of the airway. Indian J Anaesth. 2011; 55(5): 456-462, doi: 10.4103/0019-5049.89868, indexed in Pubmed: 22174461.

15. Adhikari S, Zeger W, Schmier C, et al. Pilot study to determine the utility of point-of-care ultrasound in the assessment of difficult laryngoscopy. Acad Emerg Med. 2011; 18(7): 754-758, doi: 10.1111/j.1553-2712.2011.01099.x, indexed in Pubmed: 21707828.

16. Wu J, Dong J, Ding Y, et al. Role of anterior neck soft tissue quantifications by ultrasound in predicting difficult laryngoscopy. Med Sci Monit. 2014; 20: 2343-2350, doi: 10.12659/MSM.891037, indexed in Pubmed: 25403231

17. Pinto J, Cordeiro L, Pereira C, et al. Predicting difficult laryngoscopy using ultrasound measurement of distance from skin to epiglottis. J Crit Care. 2016; 33: 26-31, doi: 10.1016/j.jcrc.2016.01.029, indexed in Pubmed: 26948251.

18. Hui CM, Tsui BC. Sublingual ultrasound as an assessment method for predicting difficult intubation: a pilot study. Anaesthesia. 2014; 69(4): 314-319, doi: 10.1111/anae.12598, indexed in Pubmed: 24641637.
19. Yao W, Wang B. Can tongue thickness measured by ultrasonography predict difficult tracheal intubation? Br J Anaesth. 2017; 118(4): 601-609, doi: 10.1093/bja/aex051, indexed in Pubmed: 28403413.

20. Petrisor C, Szabo R, Constantinescu C, et al. Ultrasound-based quantification of submandibular space compliance as predictor for difficult intubation. A prospective observational preliminary study. J Anesth. 2017; 34(Suppll 55): 11 AP08-9.

21. Ezri T, Gewürtz G, Sessler DI, et al. Prediction of difficult laryngoscopy in obese patients by ultrasound quantification of anterior neck soft tissue. Anaesthesia. 2003; 58(11): 1111-1114, indexed in Pubmed: 14616599.

22. http://www.biosoft.hacettepe.edu.tr/easyROC/ (accesed October 20th 2016)

23. Greenland KB. Airway assessment based on a three column mode of direct laryngoscopy. Anaesth Intensive Care. 2010; 38(1): 14-19, indexed in Pubmed: 20191771.

24. Huh J, Shin HY, Kim SH, et al. Diagnostic predictor of difficult laryngoscopy: the hyomental distance ratio. Anesth Analg. 2009; 108(2): 544-548, doi: 10.1213/ane.0b013e31818fc347, indexed in Pubmed: 19151285.

25. Sustić A. Role of ultrasound in the airway management of critically ill patients. Crit Care Med. 2007; 35(5 Suppl): S173-S177, doi: 10.1097/01. CCM.0000260628.88402.8A, indexed in Pubmed: 17446776.

26. Wojtczak J, Bonadonna P. Pocket mobile smartphone system for the point-of-care submandibular ultrasonography. Am J Emerg Med. 2013; 31(3): 573-577, doi: 10.1016/j.ajem.2012.09.013, indexed in Pubmed: 23159431.

27. Andruszkiewicz P, Wojtczak J, Sobczyk D, et al. Effectiveness and validity of sonographic upper airway evaluation to predict difficult laryngoscopy. JUltrasound Med. 2016; 35(10): 2243-2252, doi: 10.7863/ ultra.15.11098, indexed in Pubmed: 27582532.

28. El-Orbany M, Woehlck H, Salem MR. Head and neck position for direct laryngoscopy. Anesth Analg. 2011; 113(1): 103-109, doi: 10.1213/ ANE.0b013e31821c7e9c, indexed in Pubmed: 21596871.

29. Fulkerson JS, Moore HM, Anderson TS, et al. Ultrasonography in the preoperative difficult airway assessment. J Clin Monit Comput. 2017; 31(3): 513-530, doi: 10.1007/s10877-016-9888-7, indexed in Pubmed: 27156094.

\section{Corresponding author:}

\section{Cristina Petrisor}

Department of Anaesthesia and Intensive Care

Clinical Emergency County Hospital, Cluj

400006 Clinicilor 3-5, Cluj-Napoca, Romania

e-mail:petrisor.cristina@umfcluj.ro

Received: 15.09.2017

Accepted: 8.05.2018 